\section{Myeloid sarcoma without circulating leukemia mimicking gastrointestinal malignancy and lymphoma}

\author{
Sravanthi Ravulapati, Craig Siegel, \\ Ameesh Dara, Jack M. Lionberger \\ Division of Hematology and Oncology, \\ St. Louis University School of Medicine, \\ MO, USA
}

\begin{abstract}
We present an unusual case of myeloid sarcoma with ascites and abdominal pain in which initial clinical, laboratory, and imaging studies suggested a gastrointestinal malignancy or lymphoma. Subsequent detection of leukemic ascites and blasts in a gastric, small bowel, and skin biopsy supported a diagnosis of myeloid sarcoma. Bone marrow biopsy revealed $15 \%$ blasts, and cytogenetics with an inversion 16 rearrangement was diagnostic of acute myeloid leukemia (AML). Positron emission tomography-computed tomography performed at presentation to stage a presumptive lymphoma found later utility in following the burden of extramedullary disease. Standard AML induction chemotherapy resulted in complete remission and was followed by three rounds of high dose cytarabine consolidation. The patient unfortunately relapsed leading to re-induction followed by allogeneic stem cell transplantation. This report describes the presentation, assessment, and management of myeloid sarcoma.
\end{abstract}

\section{Introduction}

Myeloid sarcoma is characterized by abnormal myeloid blasts at extramedullary sites and rarely presents without prior or concomitant leukemia. ${ }^{1}$ Despite modern diagnostic techniques of flow cytometry and immunohistochemical staining, myeloid sarcoma arising as the primary disease site is a challenge to diagnose. Additionally without prospective trials, this rare entity has no universally agreed upon diagnostic and treatment protocols. Herein, we describe a patient with myeloid sarcoma that presented with abdominal pain and ascites initially suggesting a gastrointestinal tumor or lymphoma. The case highlights current issues pertaining to the diagnosis and management of patients with this malignancy.

\section{Case Report}

A 49-year-old Caucasian male with a medical history of hypertension and coronary artery disease with recent coronary artery stent placement presented to an outside hospital complaining of abdominal pain, night sweats and weight loss. Computed tomography (CT) of the abdomen and pelvis showed ascites, increased attenuation of the mesentery throughout the abdomen and pelvis, and a 2 $\mathrm{cm}$ liver nodule suggesting peritoneal carcinomatosis. Exploratory laparotomy revealed purulent fluid, thickened mesentery, and a granular omentum. Omental and peritoneal biopsies showed sheets of large cells suggestive of lymphoma.

The patient was transferred to Saint Louis University (SLU) Hospital. Physical exam revealed distended abdomen with ascites flowing into a surgical drain in the right lower quadrant. A $3 \times 3 \mathrm{~cm}$ subcutaneous non-tender mass on the anterior aspect of the left shoulder and a $2.5 \times 2 \mathrm{~cm}$ mass on the right chest wall were observed. Labs revealed white blood cell (WBC) count $11.7 \times 10^{3} / \mathrm{uL}$, hemoglobin $9.1 \mathrm{mg} / \mathrm{dL}$, and platelets $517 \times 10^{3} / \mathrm{uL}$. Manual differential of the WBC count showed $81 \%$ neutrophils, $6 \%$ lymphocytes, $11 \%$ monocytes, and $2 \%$ eosinophils. There were no blasts or other immature myeloid cells noted. Renal and hepatic function were normal. Uric acid was $5.1 \mathrm{mg} / \mathrm{dL}$ and lactase dehydrogenase (LDH) was $367 \mathrm{U} / \mathrm{L}$.

While the differential diagnosis included lymphoma, positron emission tomography-computed tomography (PET-CT) was performed that showed intense fluorodeoxyglucose (FDG) avidity in the peritoneum, mesentery, retroperitoneal and porta hepatis lymph nodes, as well as the two soft tissue nodules described above.

Peritoneal fluid analysis revealed a WBC of $48 \times 10^{3} / \mathrm{uL}$ with $21.8 \%$ blast cells positive for CD117, CD34, MPO, and CD43 but negative for CD30, MUM1, CD56, CD4, and TdT, a profile consistent with myeloid sarcoma (Figure 1). Upper and lower gastrointestinal endoscopy with biopsy was performed showing large immature CD34 positive blasts in the lamina propria of the stomach and small intestine. Similar cells were found on biopsy of the subcutaneous masses (Figure 2). Bone marrow biopsy demonstrated a hypercellularity and increased blasts $(10-15 \%$ by CD34 immunohistochemical staining), mild dyserythropoiesis, dysmegakaryopoiesis, and no increase in reticulin fibrosis. Cytogenetics revealed $46 \mathrm{XY}$ with an inversion on chromosome 16 (inv (16)). We
Correspondence: Jack Lionberger, Division of Hematology and Oncology, St. Louis University School of Medicine, 3rd floor, 3655 Vista Avenue, St. Louis, MO 63110, USA.

Tel.: +1.314.268.7109 - Fax: +1.314 .773 .1167$

E-mail: jlionber@slu.edu

Key words: Myeloid sarcoma; granulocytic sarcoma; acute myeloid leukemia.

Contributions: CS, SR, AD: reference search and manuscript writing, JL manuscript review and editing

Conflicts of interest: the authors declare no potential conflict of interest.

Received for publication: 10 November 2016 . Revision received: 22 February 2017.

Accepted for publication: 24 March 2017.

This work is licensed under a Creative Commons Attribution-NonCommercial 4.0 International License (CC BY-NC 4.0).

(C) Copyright C. Siegel et al., 2018

Licensee PAGEPress, Italy

Hematology Reports 2018; 10:7040

doi:10.4081/hr.2018.7040

regarded this to be consistent with the diagnosis of acute myeloid leukemia (AML) despite the blasts being less than $20 \%$.

SLU standard induction chemotherapy was delivered - cytarabine, idarubicin, and cladribine. ${ }^{2}$ Treatment resulted in decreased output from the abdominal drain and improved ascites but was complicated by neutropenic enterocolitis and vancomycin resistant enterococcus bacteremia. The patient improved, and bone marrow biopsy on day 26 showed complete remission with no leukemic blasts. Repeat PET-CT on day 47 demonstrated complete resolution of abnormal abdominal signal (Figure 3). Thereafter, the patient received three courses of high dose cytarabine consolidation, and we monitored PET-CT with each follow up bone marrow biopsy.

Eight months after induction chemotherapy, the patient developed bloating and abdominal pain similar to his initial presentation. PET-CT and bone marrow biopsy at that time were negative, and symptoms resolved spontaneously within a few days.

Sixteen months after induction, the patient developed abdominal pain and vomiting. Laboratory showed WBC of $171 \times 10^{3} / \mathrm{uL}$, and marrow biopsy confirmed relapsed AML. PET-CT was not repeated at relapse, since relapse was apparent by other clinical features. Re-induction was per- 
formed with cladribine, cytarabine, granulocyte colony stimulating factor, and mitoxantrone (CLAG-M). ${ }^{3}$ At second remission, he received a myeloablative matched unrelated donor allogeneic stem cell transplantation. As of submission of this manuscript, the patient is 6 months post-transplant with no evidence of disease recurrence.

\section{Discussion}

Myeloid sarcoma (also known as granulocytic sarcoma or chloroma) is a neoplasm characterized by abnormal myeloid cells at extramedullary sites and is an uncommon manifestation of AML. Myeloid sarcoma may present before, during, or after a diagnosis of AML or represent a transformation of a prior myeloproliferative or myelodysplastic condition. ${ }^{1}$

Our patient illustrates the diagnostic challenges associated with myeloid sarcoma. De novo presentation without previous or concurrent evidence of leukemia in the peripheral blood or bone marrow is extremely rare. In a case series from MD Anderson, primary (non-leukemic) myeloid sarcoma accounted for $1.4 \%$ of all AML over a 12 year period. ${ }^{4}$ The most common sites of involvement were skin (48\%), lymph node (33\%), testes $(23 \%)$, and CNS $(19 \%)$. Other sites included breast, bone, mesentery, and spleen. ${ }^{4}$ Historically, primary myeloid sarcoma is difficult to diagnose, because it mimics lymphomas and solid tumors. Our patient presented with extramedullary features, no peripheral blood leukemia, and less than $20 \%$ blasts in the bone marrow. His abdominal symptoms and ascites initially suggested a GI malignancy. Although current use of flow cytometry and immunohistochemical staining facilitates correct pathologic diagnosis, as in this case, misdiagnosis as an aggressive lymphoma, sarcoma, or even carcinoma occurs. ${ }^{5,6}$ In an Italian series from 2007 , $10 / 25(40 \%)$ patients with myeloid sarcoma were initially misdiagnosed as various types of lymphoma. ${ }^{5}$

PET-CT can be useful in the diagnosis and subsequent management of myeloid sarcoma. PET-CT is sensitive for revealing clinically unrecognized disease burden, or as a tool for assessing response to treatment or relapse. ${ }^{7,8}$ In one small study, PET-CT evidence of resolution in organs correlated with pathologic clearance of disease in the bone marrow. In contrast, complete response in the bone marrow with only partial response by PET-CT was associated with early relapse. ${ }^{7}$ We found PET-CT useful in excluding recurrence when our patient developed post-therapy abdominal symptoms similar to his presentation.

The cytogenetic and molecular abnormalities associated with myeloid sarcoma are similar to those seen in leukemic AML. ${ }^{5}$ However, abnormalities in chromosome 8 , in particular $\mathrm{t}(8 ; 21)$ in children and trisomy 8 are more common in myeloid sarcoma compare to leukemic AML. ${ }^{9}$ Due to small study samples, it is unclear whether this finding is valid or skewed due to a referral bias related to the younger aged population. Our patient had an inv(16), and interesting- ly this anomaly is often found in myeloid sarcoma associated with intestinal manifestations. $^{5,6}$ It is possible that the intestine may be a tissue specific target for the expression of $\mathrm{CBF} \beta / \mathrm{MYH} 11$, the fusion protein expressed by inv(16). ${ }^{6}$

Ideal treatment of myeloid sarcoma is unclear, as this patient population is small and no randomized controlled trials exist. There is controversy regarding the impact of extramedullary features on AML. 4,10 Case reports and series suggest that without systemic treatment, even isolated myeloid sarcoma will progress to acute leukemia over a short time course. ${ }^{11}$ Therefore, we agree with Blast et al. that patients with myeloid sarcoma, even in the absence of a leukemic component at diagnosis, should be treated with standard AML induction. ${ }^{12}$ Isolated myeloid sarcoma seem to respond similarly to leukemic AML in cases that share similar prognostic features. ${ }^{10}$ Radiation therapy to local sites of infiltration has been added to cases with incomplete response after systemic chemotherapy, but it is unclear if this approach is superior to chemotherapy alone. ${ }^{12}$

\section{Conclusions}

The decision to proceed to allogeneic stem cell transplantation is not specifically impacted by the presence of myeloid sarcoma. Rather, as is the case with patients without extramedullary involvement, risk assessment requires demographic, clinical,

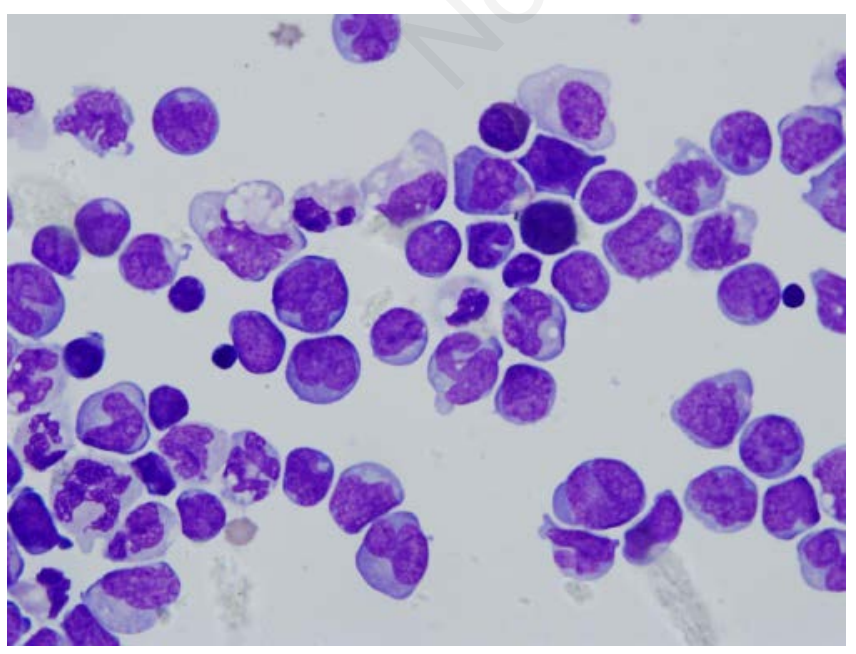

Figure 1. Cytospin of peritoneal fluid containing immature hematopoietic blasts.

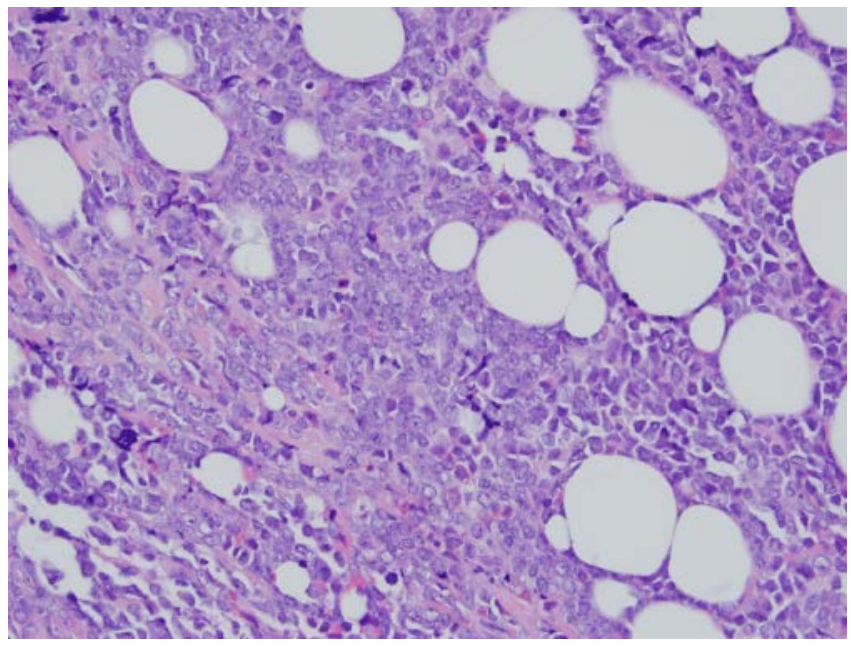

Figure 2. Left chest wall mass biopsy, presence of immature hematopoietic blasts. 


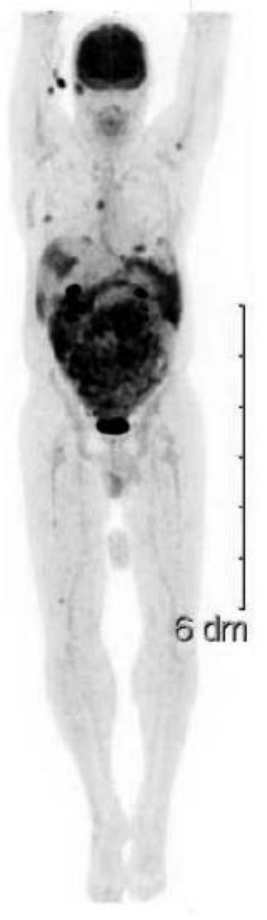

Figure 3. Pre (left) and post (right) induction therapy PET-CT scans showing complete metabolic response.

cytogenetic, and molecular features to guide the decision. ${ }^{12}$ Typically, inv(16) positive AML yields a favorable response to intensive therapy, and after discussion with the patient, we elected to defer allogeneic stem cell transplant until second complete remission.

\section{References}

1. Neiman RS, Barcos M, Berard C, et al. Granulocytic sarcoma: a clinicopathologic study of 61 biopsied cases. Cancer 1981;48:1426-37.

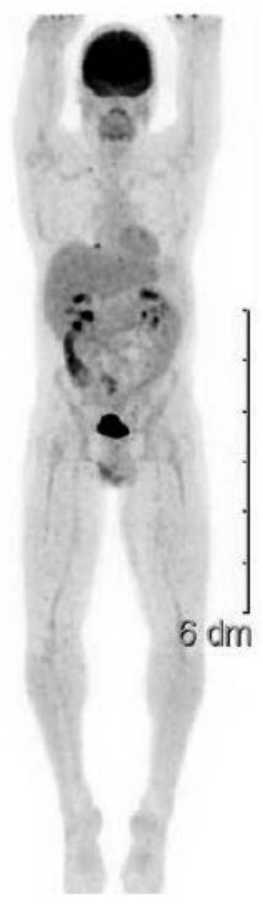

2. Holowiecki J, Grosicki S, Giebel S, et al. Cladribine, but not fludarabine, added to daunorubicin and cytarabine during induction prolongs survival of patients with acute myeloid leukemia: a multicenter, randomized phase III study. J Clin Oncol 2012;30:2441-8.

3. Wierzbowsa A, Robak T, Pluta A, et al. Cladribine combined with high doses of arabinoside cytosine, mitoxantrone, and GCSF (CLAG-M) is a highly effective salvage regimen in patients with refractory and relapsed acute myeloid leukemia of the poor risk: a final report of the Polish Adult Leukemia Group. Euro J Haematol 2008;80:115-26.
4. Tsimberidou AM, Kantarjian HM, Estey E, et al. Outcome in patients with nonleukemic granulocytic sarcoma treated with chemotherapy with or without radiotherapy. Leukemia 2003;17: 1100-3.

5. Pileri SA, Ascani S, Cox MC, et al. Myeloid sarcoma: clinic-pathologic, phenotypic and cytogenetic analysis of 92 adult patients, Leukemia 2007;21: 340-50.

6. Zhang X, Zhang R, Li, Y. Granulocytic sarcoma of abdomen in acute myeloid leukemia patient with inv(16) and t $(6 ; 17)$ abnormal chromosome: Case report and review of literature. Leukemia Research 2010;34:958-61.

7. Cribe A-SWI, Steenhof M, Marcher $\mathrm{CW}$, et al. Extramedullary disease in patients with acute myeloid leukemia assessed by (18)F-FDG PET. Eur J Hematol 2013;90:273-8.

8. Stölzel F, Röllig C, Radkeet J, et al. ${ }^{18} \mathrm{~F}$ FDG-PET/CT for detection of extramedullary acute myeloid leukemia. Haematologica 2011;96: 1552-6.

9. Byrd JC, Edenfield WJ, Shields DJ, Dawson NA. Extramedullary myeloid cell tumors in acute nonlymphocytic leukemia: a clinical review, J Clin Oncol. (1995)13, 1800-16.

10. Tsimberdou AM, Kantarjian HM, Wen $\mathrm{S}$, et al. Myeloid sarcoma is associated with superior event-free survival and overall survival compared with acute myeloid leukemia. Cancer 2008;113: 1270-8.

11. Yamauchi K, Yasuda M. Comparison in treatments of nonleukemic granulocytic sarcoma. Cancer 2002;94;1739-46

12. Blast RL, Tallman MS, Douer D, Yahalom J. How I treat extramedullary acute myeloid leukemia. Blood 2011;118:3785-93. 